\title{
Impact of Beta-Amyloid-Specific Florbetaben PET Imaging on Confidence in Early Diagnosis of Alzheimer's Disease
}

\author{
C.G. Schipke $\quad$ O. Peters ${ }^{b} \quad$ I. Heuser ${ }^{b} \quad$ T. Grimmer $\quad$ M.N.Sabbagh ${ }^{a} \quad$ O. Sabrie \\ C. Hock ${ }^{f}$ M. Kunz ${ }^{c} \quad J . K^{2}$ Kuhlmann ${ }^{c} \quad$ C. Reininger ${ }^{c} \quad$ M. Blankenburg ${ }^{c}$ \\ aBanner Sun Health Research Institute, Sun City, Ariz., USA; ${ }^{b}$ Department of Psychiatry, Charité-Campus Benjamin \\ Franklin, 'Bayer HealthCare Pharmaceuticals, Berlin, dDepartment of Psychiatry and Psychotherapy, Klinikum \\ rechts der Isar, Technische Universität München, Munich, and 'Department of Nuclear Medicine, University

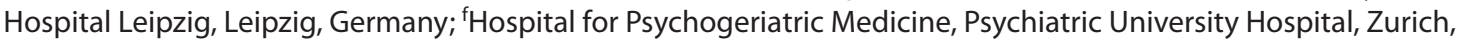 \\ Switzerland
}

\section{Key Words}

Alzheimer's disease • Beta-amyloid plaques • Florbetaben amyloid imaging

\begin{abstract}
Background: Early diagnosis of Alzheimer's disease (AD) may be corroborated by imaging of beta-amyloid plaques using positron emission tomography (PET). Here, we performed an add-on questionnaire study to evaluate the relevance of florbetaben imaging (BAY 94-9172) in diagnosis and consecutive management of probable AD patients. Methods: $A D$ patients with a clinical diagnosis in accordance with the NINCDS-ADRDA criteria or controls were imaged using florbetaben. Referring physicians were asked on a voluntary basis about their confidence in initial diagnosis, significance of PET imaging results, and their anticipated consequences for future patient care. Results: 121 questionnaires for probable $A D$ patients and 80 questionnaires for controls were evaluated. In $18 \%$ of patients who had initially received the diagnosis of probable AD, PET scans were rated negative, whereas in controls $18 \%$ of scans were positive. An increase in confidence in the initial diagnosis was frequently reported (80\%). Imaging results had a significant impact on the in-
\end{abstract}

tended patient care, as judged by the referring physicians; this was most prominent in those patients with a contradicting scan and/or a low confidence in the initial diagnosis. Conclusion: Florbetaben amyloid imaging increases the overall confidence in diagnosis of $A D$ and may frequently influence clinical decisions and patient management.

Copyright @ 2012 S. Karger AG, Basel

\section{Introduction}

Biomarkers that support early diagnosis of Alzheimer's disease $(\mathrm{AD})$ are useful in the differential diagnosis and urgently needed in disease-modifying therapeutic strategies and longitudinal monitoring of disease progression. A pivotal problem in early diagnosis of $\mathrm{AD}$ is that clinical symptoms as assessed by neuropsychological tests become apparent only after massive neuronal cell loss has already taken place. Today, there is general agreement that diagnosis of AD should be based on the results of various neuropsychological tests [1], brain imaging [2-4], cerebrospinal fluid (CSF) and blood studies [5-7],

C.G.S. and O.P. contributed equally to this work.

\begin{tabular}{lcc}
\hline KARGER & $\begin{array}{c}\text { @ 2012 S. Karger AG, Basel } \\
\text { 1420-8008/12/0336-0416\$38.00/0 Openger }\end{array}$ \\
$\begin{array}{l}\text { Fax +4161306 12 34 } \\
\text { E-Mail karger@karger.ch } \\
\text { www.karger.com }\end{array}$ & $\begin{array}{l}\text { This is an Open Access article licensed under the terms of the } \\
\text { Creative Commons Attribution-NonCommercial 3.0 Un- } \\
\text { ported license (CC BY-NC) (www.karger.com/OA-license), } \\
\text { applicable to the online version of the article only. Distribu- } \\
\text { tion permitted for non-commercial purposes only. }\end{array}$
\end{tabular}


the patient's own and family history, and a physical examination. Recently, revised guidelines for the clinical diagnosis of $\mathrm{AD}$ have been proposed by the National Institute on Aging and the Alzheimer's Association [8]. These guidelines add altered biomarkers as a criterion to enhance the likelihood of underlying AD pathology in the diagnosis of probable AD. Additionally, since a number of disease-modifying therapies like vaccination and immune-modulating strategies are under investigation [9], better diagnostic tools within the biomarker field will be valued.

An ideal biomarker reliably reflects important processes of the underlying disease pathology. In $\mathrm{AD}$, certain biomarkers become abnormal, presumably in a temporally ordered manner. According to a recently published model, at the earliest stage of $\mathrm{AD}$, changes in CSF betaamyloid appear first, reaching a plateau by the time clinical symptoms emerge [10]. Consecutively, an increase in biomarkers reflecting neuronal injury such as total- and phospho-Tau protein may be followed by first clinical symptoms. To date, beta-amyloid and Tau protein can be determined in the CSF using different techniques [11], but results may be sometimes difficult to interpret. CSF biomarker levels may be weighted by sources of error: Amyloid-beta 1-42 and total-Tau protein levels vary up to 3 -fold in different studies $[12,13]$. In addition, concentrations may differ depending on the modalities of lumbar puncture, such as time of day and amount of CSF taken [14].

Recently, compounds which can be used in positron emission tomography (PET) to directly visualize betaamyloid plaques within the human brain have come into focus as a tool to improve diagnosis of AD. The Pittsburgh compound B (PIB), an analog of thioflavin T, represents the most widely used tracer for investigational PET imaging of beta-amyloid plaques in the brain. Retention of the 11C-labeled $\mathrm{PiB}([\mathrm{C}-11] \mathrm{PiB})$ has been shown to be approximately 2 -fold greater in cortical areas of $\mathrm{AD}$ subjects relative to healthy controls, and the patterns of retention reflect the patterns of beta-amyloid deposition known from postmortem studies [15]. Florbetaben, an 18 F-labeled tracer utilizing a radioisotope with a longer half-life than PIB $[16,17]$ is currently being tested in a phase III clinical trial program.

Here, we report on a study to address the question how in a clinical setting the information of results from amyloid-PET imaging in patients with possible or probable $\mathrm{AD}$ would influence diagnostic reevaluations of physicians, their choice of treatment options and their intended general patient management.

Florbetaben PET Imaging in Early

Diagnosis of Alzheimer's Disease
Table 1. Correlation between the degree of confidence in the initial diagnosis and the level of impact on management of probable $\mathrm{AD}$ patients

\begin{tabular}{lllr}
\hline $\begin{array}{l}\text { Degree of confidence in } \\
\text { preexisting diagnosis }\end{array}$ & \multicolumn{2}{l}{$\begin{array}{l}\text { Level of impact on patient } \\
\text { management }\end{array}$} & \\
\cline { 2 - 4 } & $\begin{array}{l}\text { strong }+ \\
\text { some }\end{array}$ & $\begin{array}{l}\text { minor }+ \\
\text { none }\end{array}$ & total \\
\hline Very high & & & \\
$\quad$ Probable AD patients & 25 & 33 & 58 \\
$\quad$ Confirming scan & 24 & 30 & 54 \\
$\quad$ Contradicting scan & 1 & 3 & 4 \\
Moderate + low & & 13 & 63 \\
$\quad$ Probable AD patients & 50 & 12 & 45 \\
$\quad$ Confirming scan & 33 & 1 & 18 \\
$\quad$ Contradicting scan & 17 & 46 & 121 \\
Sum & & 42 & 99 \\
$\quad$ Probable AD patients & 75 & 4 & 22 \\
$\quad$ Confirming scan & 57 & & \\
Contradicting scan & 18 & &
\end{tabular}

Fisher exact test (d.f. $=1)$. Probable AD patients: $\chi^{2} 15.35 ; \mathrm{p}$ value 0.00009 ; odds ratio $0.20, \mathrm{CI}_{95}$ 0.09-0.44. Confirming scans: $\chi^{2} 7.25$; $\mathrm{p}$ value 0.007 ; odds ratio $0.29, \mathrm{CI}_{95} 0.12-0.68$. Contradicting scans: $\chi^{2} 6.45$; $\mathrm{p}$ value 0.011 ; odds ratio $0.02, \mathrm{CI}_{95} 0.00-0.41$.

\section{Methods}

\section{Participants}

Between February 2010 and March 2011, a questionnaire study was conducted as an add-on to the multicenter beta-amyloid-specific florbetaben PET phase IIb trial with a total of 272 patients (demographic data in online suppl. table 1; for all online suppl. material, see www.karger.com/ doi/10.1159/000339367). Study participants were either patients diagnosed with probable AD or healthy volunteers that were directly recruited as unimpaired controls from the community. The lack of cognitive impairment in healthy volunteers was based on an MMSE result $\geq 28$ and a CDR of 0 . Patients with probable AD had to fulfill both the DSM-IV criteria for dementia of $\mathrm{AD}$ and the NINCDS-ADRDA criteria for AD. The MMSE score had to be between 18 and 26 and the CDR 0.5 , 1.0 or 2.0. Duration and history of the disease varied between probable AD patients. CSF biomarker values were only available for a small subset of patients, were not part of the study protocol, and were not further evaluated. For both groups, the diagnosis had been affirmed by magnetic resonance brain imaging before admission to PET scan.

The questionnaire was given to referring physicians in Australia, Germany, Japan, Switzerland, and USA whose patients received florbetaben PET imaging in the context of the phase IIb trial. Fourteen out of a total of 20 centers participated on a voluntary basis. A total of 201 questionnaires were completed and sent back to the authors (supplementary table 2).

Dement Geriatr Cogn Disord 2012;33:416-422 
Table 2. Analysis of homogeneity amongst the 14 participating centers

\begin{tabular}{lcc}
\hline Questionnaire item and group & $\begin{array}{c}\text { Value of the } \\
\text { test statistic }\end{array}$ & $\begin{array}{l}\mathrm{p} \\
\text { value }\end{array}$ \\
\hline Confidence in diagnosis before PET scan & \\
AD, all results & 8.5693 & 0.0034 \\
AD, negative scan result & 0.6341 & 0.4259 \\
AD, positive scan result & 10.5393 & 0.0012 \\
UC, all results & 3.5680 & 0.0589 \\
UC, negative scan result & 12.3381 & 0.0004 \\
UC, positive scan result & 2.3257 & 0.1273 \\
\hline Change of confidence in diagnosis after PET scan & \\
AD, all results & 8.0577 & 0.0028 \\
AD, negative scan result & $n . v$. & $n . v$. \\
AD, positive scan result & 8.2022 & 0.0042 \\
UC, all results & 0.2456 & 0.6202 \\
UC, negative scan result & 2.5937 & 0.1073 \\
UC, positive scan result & 10.5670 & 0.0012 \\
\hline Impact on patient management after PET scan & \\
AD, all results & 0.0248 & 0.8748 \\
AD, negative scan result & 1.9050 & 0.1675 \\
AD, positive scan result & 0.0237 & 0.8776 \\
UC, all results & 0.1591 & 0.6899 \\
UC, negative scan result & 9.2785 & 0.0023 \\
UC, positive scan result & 7.3014 & 0.0069 \\
& &
\end{tabular}

Cochran-Mantel-Haenszel test; $\mathrm{p}<0.05$, indicative of heterogeneity; $\mathrm{p}>0.05$, not indicative of heterogeneity; $\mathrm{AD}=$ probable $\mathrm{AD}$ patients; $\mathrm{UC}=$ unimpaired controls; n.v. = no variability.

\section{Procedure}

A face-validated, 'fit-for-purpose' clinician questionnaire was designed, consisting of six items evaluating (1) the level of confidence in the initial clinical diagnosis before PET imaging results were known, (2) the objective results of the florbetaben PET scan, (3) the congruency of the PET imaging and the initial diagnosis, (4) the potential change of confidence in the overall diagnosis on the basis of the PET scan results, (5) the importance, and (6) the characteristics of the potential impact on patient management. For the original questionnaire, please refer to the supplementary material.

The questionnaire was validated in advance for clarity and appropriateness by clinical physicians. The questionnaires were completed by the clinical centers that were referring patients to the respective nuclear medicine departments. The returned questionnaires had been fully anonymized.

\section{Statistical Analysis}

For statistical analysis, SAS version 9.1 was used. Results are expressed as number (\%) or means \pm SD unless indicated otherwise. Frequency distributions for categorical variables (see fig. 4) were compared using the Fisher exact test (table $1)$.

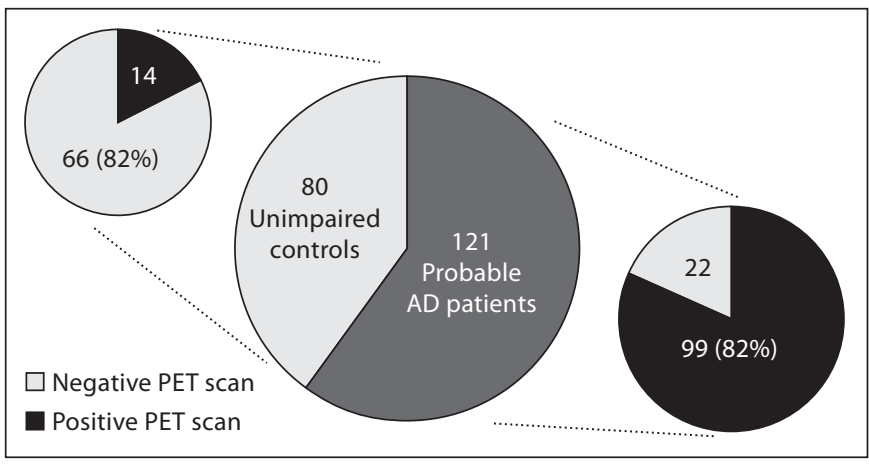

Fig. 1. Frequency of positive and negative florbetaben PET scan results. Numbers are given for patients with the clinical diagnosis of probable $\mathrm{AD}$ and for unimpaired controls.

Homogeneity of the participating centers (table 2) was analyzed using the Cochran-Mantel-Haenszel test stratifying for centers. The variables collected were dichotomized as follows: the item 'confidence in diagnosis before PET scan' into 'high confidence' and 'moderate + weak + very weak confidence'; the item 'change of confidence in diagnosis after PET scan' into 'increased + decreased confidence' and 'unchanged confidence'; the item 'impact on patient management after PET scan' into 'strong + some impact' and 'minor + no impact'.

\section{Results}

Fourteen out of the 20 centers participating in the florbetaben phase IIb clinical trial returned 201 questionnaires (74\% response rate): 121 referred to probable $\mathrm{AD}$ patients and 80 to unimpaired controls. Among the 121 probable AD patients, 22 (18\%) patients showed no significant beta-amyloid plaque load as examined using the onsite beta-amyloid plaque load visual assessment score [17]. On the other hand, $18 \%(n=14$ out of 80$)$ of the controls presented with a significant plaque load as assessed by the onsite nuclear physician (fig. 1).

\section{After Knowledge of PET Outcome, Change of \\ Confidence in Diagnosis Is Common}

Focusing on $\mathrm{AD}$ patients, we were interested to learn whether the results of the beta-amyloid-specific PET scan lead to a change of diagnostic confidence. The overall change of confidence in the initial diagnosis after receiving the PET scan results was common: in $83 \%$ of all probable $\mathrm{AD}$ cases, a change of confidence, either an increase or a decrease, in the original diagnosis was reported $(\mathrm{n}=22+77+2=101$ of 121 ; black and light 


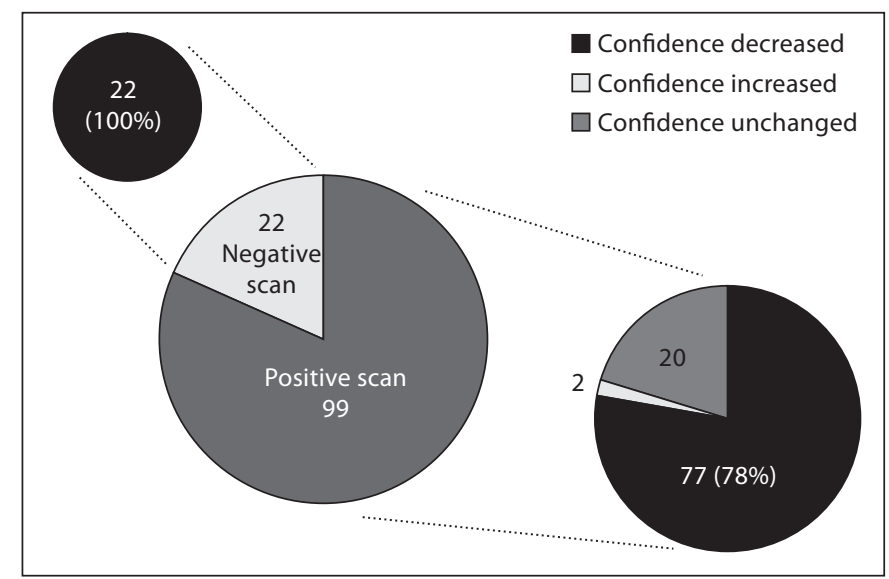

Fig. 2. Frequency and direction of change of confidence in the initial diagnosis on receiving the result of the florbetaben PET scan. Numbers are given for the 121 probable AD patients.

grey areas, fig. 2). In all probable $\mathrm{AD}$ cases with a negative PET scan $(n=22)$, physicians reported a decrease in confidence in their original diagnosis (fig. 2). In probable AD cases with an expected positive PET scan result, the physicians indicate in $78 \%$ of the cases an increase ( $\mathrm{n}$ $=77)$, in $2 \%$ a decrease $(\mathrm{n}=2$; reportedly due to lower signal intensities in the PET scans than expected by the physicians), and in $20 \%$ no change of confidence in their initial diagnosis $(\mathrm{n}=20)$.

Even in the group of controls, a change of confidence in the initial clinical evaluation was reported in $43 \%$ of all the cases ( $n=34$ out of 80 ; data not shown). Within the group of controls with an unexpected positive scan, 10 out of 14 cases $(71 \%)$ reported a decrease in confidence in the original diagnosis; within the group of controls with an expected negative scan, 24 out of 66 cases (36\%) reported an increase in confidence.

\section{Physicians Anticipate Impact on Patient Management}

In a second step, we aimed to determine whether the results of the PET scan could have an influence on patient management. Overall, physicians reported an anticipated impact on their patient management of probable AD patients in $89 \%$ of the cases $(n=108$ out of 121$)$ and in $35 \%$ of the controls $(n=28$ out of 80$)$. Regarding the impact level (strong, some, minor, no impact), we detected a wide range ( $\mathrm{n}=35$ vs. 40 vs. 33 vs. 13 cases, respectively; fig. 3 ), and in about 2 out of 3 probable $\mathrm{AD}$ patients either a strong or some impact. In controls, this distribution was more pronounced ( $\mathrm{n}=1$ vs. 17 vs. 10 vs.

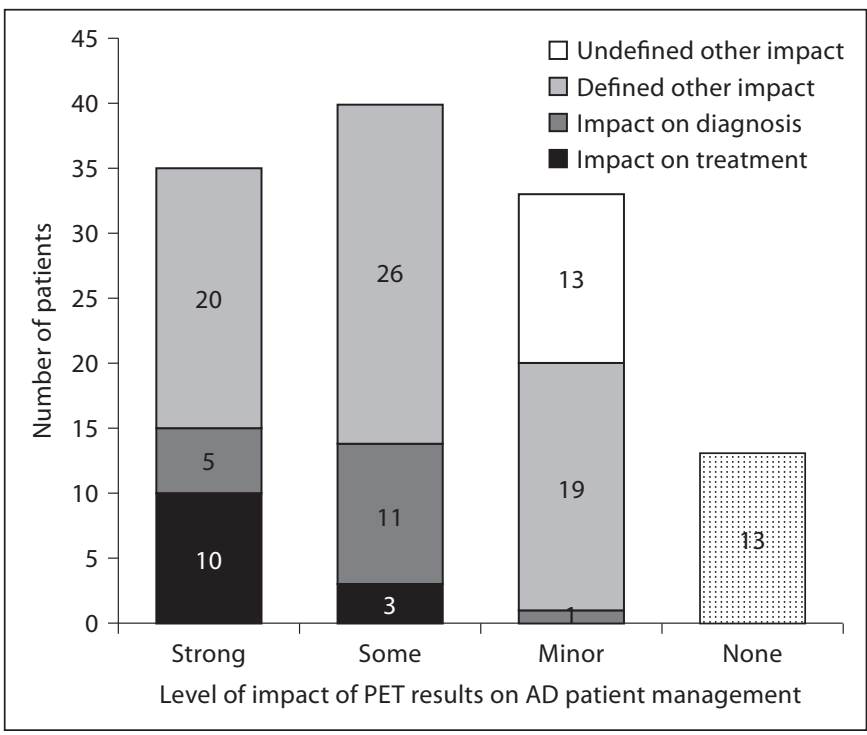

Fig. 3. Level and type of impact on patient management in probable AD patients. For the 121 probable AD patients, the frequency of strong, some, minor, and no impact reported by the physicians is depicted in the four columns. The various shades of the columns indicate the type of impact. Note that although all these patients had been diagnosed with probable $\mathrm{AD}$ before receiving a PET scan for beta-amyloid, there is a remarkable number of patients with a significant impact reported.

52 cases, respectively; not shown). The actual measures and changes in patient management at a later time point were not evaluated within this study.

When further dissecting the characteristics of the impact in cases of probable $\mathrm{AD}$, we frequently found for 'strong impact' and 'some impact' a relevance regarding the intended treatment or alternative diagnostic options (fig. 3, black and dark grey areas). The term 'defined other impact' in strong, some and minor impact categories includes 'reassurance to continue the preexisting treatment', 'recommendations for a modified lifestyle', 'change in communication with the patient', and occasionally 'validation for inclusion into a vaccination trial' (fig. 3, light grey areas). A minor impact with no further specification was reported in 13 patients (fig. 3, white area in the column 'minor').

\section{Level of Impact Correlates with Degree of Confidence} in NINCDS-ADRDA-Based Diagnosis

To understand what renders results of amyloid-specific PET scans having a potentially high or a low clinical impact, we tested for a possible correlation between the degree of impact and the level of confidence in the origi- 


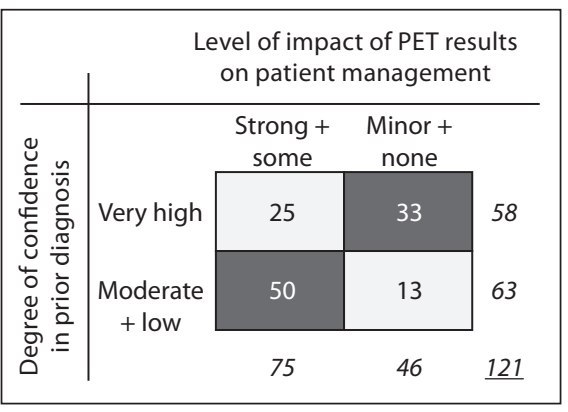

Fig. 4. Confidence in the initial diagnosis versus impact on patient management in the 121 probable $\mathrm{AD}$ patients. The numerics in the contingency table represent the numbers of the combined groups. The numbers in italics represent the sums across the respective rows and columns. Refer to table 1 for statistical analysis.

nal diagnosis of all probable $\mathrm{AD}$ cases. In this analysis, we dichotomized the categories impact and confidence and merged the answers 'strong impact/some impact', 'minor impact/no impact', and 'moderate confidence/low confidence. The frequencies of cases in the resulting three categories and the fourth category 'very high confidence' were compiled in a $2 \times 2$ contingency table as shown in figure 4. Considering the subgroups of confirming and contradicting PET scan results, we found a significant correlation using the Fisher exact test (table 1). The level of the anticipated impact is profoundly influenced by the degree of confidence in the preexisting diagnosis. This correlation is strongest in the group of preexisting $\mathrm{AD}$ diagnosis with a contradicting, negative, PET scan result (odds ratio 0.02; p value 0.011). Moreover, this effect can also be observed in probable $\mathrm{AD}$ cases where the initial diagnosis was confirmed by the PET scan result (odds ratio 0.29 ; $\mathrm{p}$ value 0.007 ).

\section{Analysis of Consistency in Reporting}

To test whether the participating physicians show center-specific variability in their judgments, we performed a statistical analysis of the homogeneity of their reporting to the questions about (1) the degree of confidence in original diagnosis, (2) the change of confidence in original diagnosis, and (3) the impact on patient management after PET scan results have been communicated (table 2). For reasons of simplicity, we combined the two categories 'decrease in confidence/increase in confidence' into a single category 'change of confidence'.

The 14 participating centers were only in partial agreement with regard to the diagnostic and therapeutic potential of amyloid imaging. Most physicians reported a change of confidence in their initial diagnosis when receiving a contradicting, negative, PET scan result. The physicians, however, varied with respect to their inclination to report a change of confidence in their original diagnosis when receiving positive PET results, both for probable $\mathrm{AD}$ patients and for controls. No significant variability amongst the centers was found for the estimated impact on patient management, irrespective of whether the PET scan results were substantiating or contradicting the initial diagnosis.

\section{Discussion}

Within the past decade, amyloid imaging was more or less restricted to highly specialized research centers because the only available tracer, the Pittsburg compound (PIB), contains the short-lived 11-carbon nuclide which has to be produced with a cyclotron onsite. With the advent of 18-fluorine-based tracers with longer half-lives amyloid-imaging will become more broadly available within the next years, and will potentially constitute an essential part of routine diagnostic procedures in $\mathrm{AD}$.

Therefore, we here evaluated the impact and usefulness of beta-amyloid-specific PET imaging using the 18F-labelled tracer florbetaben [16]. Physicians were asked about their confidence in clinical diagnosis before and after the PET results were known. Our analysis focuses on the evaluation of probable $\mathrm{AD}$ cases, since the use of beta-amyloid PET imaging in controls presently does not reflect clinical routine.

\section{Florbetaben PET Contributes to the Overall \\ Confidence in Clinical Diagnosis}

The added value of beta-amyloid-specific PET scans within the clinical routine of physicians has not been assessed so far. Not surprisingly, negative PET scans showing no amyloid burden in demented patients classified in advance as probable AD by DSM-IV and NINCDSADRDA criteria, resulted in a decreased confidence in the original diagnosis in all cases. However, in as many as $80 \%$ of probable $\mathrm{AD}$ cases when diagnosis was confirmed by a positive PET scan, results were also judged to change the confidence in the initial diagnosis. This high percentage appears unexpected, especially if one takes into account that the evaluation was conducted in specialized centers well familiar with the clinical diagnosis of AD. This finding might reflect the uncertainty of nonbiomarker NINCDS-ADRDA criteria-based AD diagno- 
sis and indicate the added value of the amyloid-specific PET scan as a method to directly visualize a patient's amyloid burden in vivo.

\section{Anticipated Treatment Regime Changes with Confidence in the Diagnosis}

One might argue that a change, i.e. a decrease or increase, of confidence in the original diagnosis has no added value for clinical physicians per se and will especially not have further implications in terms of treatment options. Our data, however, indicate that the intended treatment regime apparently changes for probable $\mathrm{AD}$ patients. Possible changes that were described included continuation of antidementive treatment, recommendation of lifestyle changes (i.e. increase in physical and social activity, etc.), and altered communication with the patient, all most likely reflecting a stronger confidence of the physicians in their actions. This might in turn contribute to a higher acceptance of and adherence to the recommended treatment amongst patients and their relatives.

In this respect, the benefit is rated highest for those cases where physicians have substantial doubts in their initial diagnosis. As this likely includes patients at early stages of their disease, one can hypothesize that they would benefit from an earlier start of drug treatment. Further, the increased confidence in diagnosis could also influence the communication and reassurance between specialist physician and primary care physician who cares for the patient in an outpatient/routine setting. Also, on the basis of the PET scan results, some patients of this study might have been included in ongoing clinical trials with beta-amyloid antibodies or other potentially disease-modifying drugs. This reflects another aspect and particular value, since a better diagnosis in terms of a quantifiable biomarker means a more meaningful evaluation of the treatment options [18]. CSF biomarker analysis was performed only in a subset of patients and was not part of the study protocol. The envisaged correlation of the PET scan and CSF biomarker results will further define the value of different diagnostic methods.
Negative PET Scans in Probable AD Prompting

Further Investigation

Our study shows negative PET scans in $18 \%$ of the probable $\mathrm{AD}$ cases that had been identified by applying the DSM-IV and the NINCDS-ADRDA criteria. This could be interpreted as a limitation of the clinical assessment on the basis of which the AD patients have been identified and included in the phase IIb study [16]. Postmortem neuropathological examination which is now underway for florbetaben in a phase III trial may clarify whether this interpretation reflects reality. To date, the correspondence of the florbetaben PET signal to the beta-amyloid plaque burden in the brain has not been described. But, the correlation of amyloid beta aggregates in frontal cortical biopsy specimen to a higher uptake of the PET tracer [11C] $\mathrm{PiB}$ has clearly been demonstrated [19].

As to the $18 \%$ positive PET scans in the unimpaired controls, it is almost certain that these individuals while having already beta-amyloid deposits in their brain do not, or not yet, suffer from cognitive deficits, which is in line with the temporal cascade concept recently reviewed [10]. Furthermore, studies that compare postmortem histopathological analyses with results of PET scans performed with the beta-amyloid tracer PiB did not yield any false-positive results [20].

Taken together, this questionnaire study shows that AD diagnosis supported by florbetaben PET imaging may increase confidence of clinicians and may influence patient management. In the context of the recently revised guidelines for the clinical diagnosis of $\mathrm{AD}$ [8], which for the first time incorporate altered biomarkers of the underlying disease state as a supportive criterion for the diagnosis $\mathrm{AD}$, the push for a stronger focus on biomarkers in the diagnosis of $\mathrm{AD}$ is evident.

\section{Acknowledgements}

We thank all coworkers in the participating centers. Also, we would like to thank all patients, their caregivers, and the healthy volunteers who participated in this study.

\section{References}

1 Wagner M, Wolf S, Reischies FM, Daerr M, Wolfsgruber S, Jessen F, Popp J, Maier W, Hull M, Frolich L, Hampel H, Perneczky R, Peters O, Jahn H, Luckhaus C, Gertz HJ, Schroder J, Pantel J, Lewczuk P, Kornhuber J, Wiltfang J: Biomarker validation of a cued recall memory deficit in prodromal Alzheimer disease. Neurology 2012;78:379-386.
2 Degerman Gunnarsson M, Lindau M, Wall A, Blennow K, Darreh-Shori T, Basu S, Nordberg A, Larsson A, Lannfelt L, Basun H, Kilander L: Pittsburgh compound-B and Alzheimer's disease biomarkers in CSF, plasma and urine: an exploratory study. Dement Geriatr Cogn Disord 2010;29:204-212.
Florbetaben PET Imaging in Early

Diagnosis of Alzheimer's Disease
Kaw Vanmechelen E: Combination of the different biological markers for increasing specificity of in vivo Alzheimer's testing. J Neural Transm Suppl 1998;53: 223-235. 
$>4$ Teipel SJ, Peters O, Heuser I, Jessen F, Maier W, Froelich L, Arlt S, Hull M, Gertz HJ, Kornhuber J, Wiltfang J, Thome J, Rienhoff O, Meindl T, Hampel H, Grothe M: Atrophy outcomes in multicentre clinical trials on Alzheimer's disease: effect of different processing and analysis approaches on sample sizes. World J Biol Psychiatry 2011;12(suppl 1): 109-113.

5 Lewczuk P, Esselmann H, Bibl M, Beck G, Maler JM, Otto M, Kornhuber J, Wiltfang J: Tau protein phosphorylated at threonine 181 in CSF as a neurochemical biomarker in Alzheimer's disease: original data and review of the literature. J Mol Neurosci 2004;23: 115-122.

$\checkmark 6$ Lewczuk P, Kamrowski-Kruck H, Peters O, Heuser I, Jessen F, Popp J, Burger K, Hampel H, Frolich L, Wolf S, Prinz B, Jahn H, Luckhaus C, Perneczky R, Hull M, Schroder J, Kessler H, Pantel J, Gertz HJ, Klafki HW, Kolsch H, Reulbach U, Esselmann H, Maler JM, Bibl M, Kornhuber J, Wiltfang J: Soluble amyloid precursor proteins in the cerebrospinal fluid as novel potential biomarkers of Alzheimer's disease: a multicenter study. Mol Psychiatry 2010;15:138-145.

$>7$ Schipke CG, Jessen F, Teipel S, Luckhaus C, Wiltfang J, Esselmann H, Frolich L, Maier W, Ruther E, Heppner FL, Prokop S, Heuser I, Peters O: Long-term stability of Alzheimer's disease biomarker proteins in cerebrospinal fluid. J Alzheimers Dis 2011;26: 255-262.

>8 Jack CR Jr, Albert MS, Knopman DS, McKhann GM, Sperling RA, Carrillo MC, Thies $\mathrm{B}$, Phelps $\mathrm{CH}$ : Introduction to the recommendations from the National Institute on Aging-Alzheimer's Association workgroups on diagnostic guidelines for Alzheimer's disease. Alzheimers Dement 2011;7:257-262.

$>9$ Blennow K, HampelH, Weiner M,Zetterberg $\mathrm{H}$ : Cerebrospinal fluid and plasma biomarkers in Alzheimer disease. Nat Rev Neurol 2010;6:131-144.
10 Jack CR Jr, Knopman DS, Jagust WJ, Shaw LM, Aisen PS, Weiner MW, Petersen RC, Trojanowski JQ: Hypothetical model of dynamic biomarkers of the Alzheimer's pathological cascade. Lancet Neurol 2010;9: 119-128.

11 Schipke CG, Prokop S, Heppner FL, Heuser I, Peters O: Comparison of immunosorbent assays for the quantification of biomarkers for Alzheimer's disease in human cerebrospinal fluid. Dement Geriatr Cogn Disord 2011; 31:139-145.

12 Shaw LM, Vanderstichele H, Knapik-Czajka M, Clark CM, Aisen PS, Petersen RC, Blennow K, Soares H, Simon A, Lewczuk P, Dean R, Siemers E, Potter W, Lee VM, Trojanowski JQ: Cerebrospinal fluid biomarker signature in Alzheimer's disease neuroimaging initiative subjects. Ann Neurol 2009;65: 403-413.

13 Mattsson N, Andreasson U, Persson S, Arai $\mathrm{H}$, et al: The Alzheimer's Association external quality control program for cerebrospinal fluid biomarkers. Alzheimers Dement 2011; 7:386-395.e386.

14 Schoonenboom NS, Mulder C, Vanderstichele H, Van Elk EJ, Kok A, Van Kamp GJ, Scheltens P, Blankenstein MA: Effects of processing and storage conditions on amyloid beta (1-42) and tau concentrations in cerebrospinal fluid: implications for use in clinical practice. Clin Chem 2005;51:189-195.

15 Wang Y, Klunk WE, Debnath ML, Huang GF, Holt DP, Shao L, Mathis CA: Development of a PET/SPECT agent for amyloid imaging in Alzheimer's disease. J Mol Neurosci 2004;24:55-62.

16 Barthel H, Gertz HJ, Dresel S, Peters O, Bartenstein P, Buerger K, Hiemeyer F, Wittemer-Rump SM, Seibyl J, Reininger C, Sabri O: Cerebral amyloid-beta PET with florbetaben $(18 \mathrm{~F})$ in patients with Alzheimer's disease and healthy controls: a multicentre phase 2 diagnostic study. Lancet Neurol 2011;10:424-435.
17 Barthel H, Luthardt J, Becker G, Patt M, Hammerstein E, Hartwig K, Eggers B, Sattler B, Schildan A, Hesse S, Meyer PM, Wolf H, Zimmermann T, Reischl J, Rohde B, Gertz HJ, Reininger C, Sabri O: Individualized quantification of brain beta-amyloid burden: results of a proof of mechanism phase 0 florbetaben PET trial in patients with Alzheimer's disease and healthy controls. Eur J Nucl Med Mol Imaging DOI: 10.1007/ s00259-011-1821-1.

18 Kornhuber J, Schmidtke K, Frolich L, Perneczky R, Wolf S, Hampel H, Jessen F, Heuser I, Peters O, Weih M, Jahn H, Luckhaus C, Hull M, Gertz HJ, Schroder J, Pantel J, Rienhoff O, Seuchter SA, Ruther E, Henn F, Maier W, Wiltfang J: Early and differential diagnosis of dementia and mild cognitive impairment: design and cohort baseline characteristics of the German DementiaCompetenceNetwork.DementGeriatr Cogn Disord 2009;27:404-417.

19 Leinonen V, Alafuzoff I, Aalto S, Suotunen T, Savolainen S, Nagren K, Tapiola T, Pirttila T, Rinne J, Jaaskelainen JE, Soininen H, Rinne JO: Assessment of beta-amyloid in a frontal cortical brain biopsy specimen and by positron emission tomography with carbon 11-labeled Pittsburgh compound B. Arch Neurol 2008;65:1304-1309.

20 Ikonomovic MD, Klunk WE, Abrahamson EE, Mathis CA, Price JC, Tsopelas ND, Lopresti BJ, Ziolko S, Bi W, Paljug WR, Debnath ML, Hope CE, Isanski BA, Hamilton RL, DeKosky ST: Post-mortem correlates of in vivo PIB-PET amyloid imaging in a typical case of Alzheimer's disease. Brain 2008;131: 1630-1645. 


\section{Erratum}

Dementia

and Geriatric

Cognitive Disorders
Figure 2 of the article 'Impact of Beta-Amyloid-Specific Florbetaben PET Imaging on Confidence in Early Diagnosis of Alzheimer's Disease' by Schipke et al. [Dement Geriatr Cogn Disord 2012; 33: 416-422 (DOI: 10.1159/000339367)] has been published with an error. Please see below the correct figure:

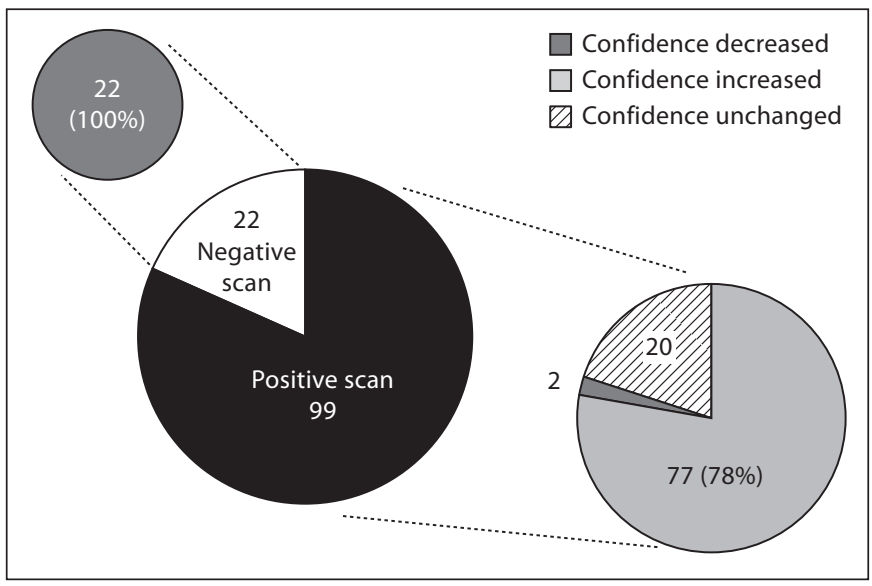

Fig. 2. Frequency and direction of change of confidence in the initial diagnosis on receiving the result of the florbetaben PET scan. Numbers are given for the 121 probable AD patients. 